\title{
Extinction of solutions of semilinear higher order parabolic equations with degenerate absorption potential
}

\author{
Y. Belaud, A. Shishkov
}

\begin{abstract}
We study the first vanishing time for solutions of the Cauchy-Dirichlet problem to the semilinear $2 m$-order $(m \geq 1)$ parabolic equation $u_{t}+L u+a(x)|u|^{q-1} u=0,0<q<1$ with $a(x) \geq 0$ bounded in the bounded domain $\Omega \subset \mathbb{R}^{N}$. We prove that if $N>2 m$ and $\int_{0}^{1} s^{-1} \operatorname{meas}\{x \in \Omega:|a(x)| \leq s\}^{\frac{2 m}{N}} d s<+\infty$, then the solution $u$ vanishes in a finite time. When $N=2 m$, the condition becomes $\int_{0}^{1} s^{-1}(\operatorname{meas}\{x \in \Omega:|a(x)| \leq s\})(-\ln \operatorname{meas}\{x \in \Omega:|a(x)| \leq s\}) d s<+\infty$.
\end{abstract}

Key words. nonlinear equation, energy method, vanishing solutions, semi-classical analysis MSC 35B40, 35K20, 35P15

\section{Introduction and main results}

Let $\Omega \subseteq \mathbb{R}^{N}, N \geq 1$, be arbitrary bounded domain. In cylindrical domain $\Omega \times(0, \infty)$ we consider the following Cauchy-Dirichlet problem

(1.1) $u_{t}+L(u)+a(x) f(u)=0$ in $\Omega \times(0, \infty), f(u)=f_{1}(u):=|u|^{q-1} u, 0<q<1$,

(1.2) $D_{x}^{\alpha} u(x, t)=0$ on $\partial \Omega \times(0, \infty), \forall \alpha:|\alpha| \leq m-1$,

(1.3) $u(x, 0)=u_{0}(x) \in L^{2}(\Omega)$.

Here $L$ is a divergent differential $2 m$-order operator of the form :

(1.4) $L(u)=(-1)^{m} \sum_{|\alpha|=m} D_{x}^{\alpha} a_{\alpha}\left(x, u, D_{x} u, \ldots, D_{x}^{m} u\right), \quad m \geqslant 1$,

with Caratheodory functions $a_{\alpha}(x, \xi)$ (continuous with respect $\xi$ and measurable with respect to $x$ ) satisfying sublinear growth condition: 


$$
\left|a_{\alpha}(x, \xi)\right| \leqslant c \sum_{|\gamma|=m}\left|\xi_{\gamma}\right| \quad \forall \xi=\left\{\xi_{\gamma}\right\} \in \mathbb{R}^{M(m)}, \quad|\alpha| \leqslant m, x \in \bar{\Omega} ; c=\text { const }
$$

where $M(m)$ is the number of different multi-indices $\gamma=\left(\gamma_{1}, \gamma_{2}, \ldots \gamma_{N}\right)$ of the length $|\gamma|:=\gamma_{1}+\cdots+\gamma_{N} \leqslant m$, and the absorptional potential $a(x)$ is nonnegative, measurable, bounded function in $\Omega$.

Our main condition on the operator $L$ is the following coercivity condition:

$$
\begin{aligned}
&(L(v), v):=\int_{\Omega} \sum_{|\alpha|=m} a_{\alpha}\left(x, v, \ldots, D_{x}^{m} v\right) D_{x}^{\alpha} v d x \geqslant C \int_{\Omega}\left|D_{x}^{m} v\right|^{2} d x \\
& \forall v \in W_{0}^{m, 2}(\Omega), C=\text { const }>0,
\end{aligned}
$$

where $W_{0}^{m, 2}(\Omega)$ is the closure in the norm $W_{0}^{m, 2}(\Omega)$ of the space $C_{0}^{m}(\Omega)$.

Remark 1.1 Well known sufficient condition of (1.6) is

$$
\sum_{|\alpha|=m} a_{\alpha}(x, \xi) \xi_{\alpha} \geqslant C \sum_{|\gamma|=m}\left|\xi_{\gamma}\right|^{2} \quad \forall \xi=\left\{\xi_{\beta}\right\} \in \mathbb{R}^{M(m)}, \quad \forall x \in \bar{\Omega} .
$$

Remark 1.2 In the linear case for operator $L=\sum_{|\alpha|=|\beta|=m} D^{\alpha} a_{\alpha \beta} D^{\beta}$ with constant coefficients $a_{\alpha \beta}$ as it is easy to check by using of Plancherel theorem, property (1.6) is guaranteed by ellipticity condition:

$$
\sum_{|\alpha|=|\beta|=m} a_{\alpha \beta} \zeta^{\alpha} \zeta^{\beta} \geqslant C|\zeta|^{2 m} \quad \forall \zeta=\left(\zeta_{1}, \ldots \zeta_{N}\right) \in \mathbb{R}^{N}, \zeta^{\alpha}:=\zeta^{\alpha_{1}} \zeta^{\alpha_{2}} \ldots \zeta^{\alpha_{N}}
$$

Definition 1.1 We will say that problem (1.1)-(1.3) has the extinction in finite time (EFT) property if for arbitrary solution $u$, there exists some positive $T_{0}$ such that $u(x, t)=0$ a.e. in $\Omega, \forall t \geq T_{0}$.

Firstly EFT-property for simplest semilinear heat equation with strong absorption was observed by A. S. Kalashnikov [4]. Later mentioned property (conditions of occurence of extinction, estimates of extinction time, asymptotic of solution near to the extinction time and so on) was investigated for different classes of second order semilinear and quasilinear parabolic equations of diffusion-absorption type by many authors (see [6,9$12,18,19])$. F. Bernis [20] proved the EFT-property for energy solutions to higher order semilinear and quasilinear parabolic equations with strong absorption. Dependence of extinction properties of energy solutions to mentioned higher order equations on local structure of initial function was studied in [14]. Extinction properties for second order semilinear parabolic equations of diffusion-absorption type with nondegenerate $(x, t)$ dependent absorptional potential was studied in [5, 7, 21,22].

V. Kondratiev, L. Veron [1] firstly initiated the study of EFT-property for second order equation (1.1) $(m=1)$ in the case of degenerate absorptional potential $a(x)$ :

$$
\inf \{a(x): x \in \Omega\}=0 .
$$


It happens that occurence of mentioned property depends essentially on the structure of the set of degeneration and on the behaviour of potential $a(x)$ in the neighbourhood of this set. They in [1] considered homogeneous Neumann problem for second order equation (1.1) $(m=1)$ and proved the following general sufficient condition for EFTproperty:

$$
\sum_{i=1}^{\infty} \mu_{i}^{-1}<\infty
$$

$$
\mu_{k}:=\inf \left\{\int_{\Omega} \sum_{i, j=1}^{N}\left(a_{i j} v_{x_{i}} v_{x_{j}}+2^{k} a(x) v^{2}\right) d x: v \in W^{1,2}(\Omega), \int_{\Omega} v^{2} d x=1\right\}, \quad \forall k \geqslant 1 .
$$

Method from [1] (semiclassical or KV-method) was developed in [2] and the following explicit sufficient condition of EFT-property for Dirichlet and Neumann boundary problem for second order equation (1.1) was established:

(1.11) $\ln a(x)^{-1} \in L^{p}(\Omega)$ for some $p>\frac{N}{2}$.

As a consequence, if $\{0\} \in \Omega$, then arbitrary potential

(1.12) $a(x): a(x) \geq a_{\alpha}(|x|):=\exp \left(-\frac{1}{|x|^{\alpha}}\right) \forall x \in \Omega$

satisfies condition (1.11) by arbitrary $\alpha<2$. On the other hand, for potential $a_{\alpha}(x)$ with $\alpha>2$ EFT-property fails [2].

In [3] there was elaborated the adaptation of local energy method from $[8,14]$ to the study of extinction properties of energy solution to second order parabolic equations with radial degenerate absorptional potential. As result the following sharp Dini-like sufficient condition of EFT-property was obtained:

$$
a(x) \geq \exp \left(-\frac{\omega(|x|)}{|x|^{2}}\right), \text { where } \omega(s)>0 \forall s>0: \omega(0)=0, \int_{0}^{1} \frac{\omega(s)}{s} d s<\infty .
$$

The drawback of using regularizing effects does not enable the KV-method to be extended to higher order operators but for small dimensions (continuous injection of $W^{m, 2}(\Omega)$ into $\left.L^{\infty}(\Omega)\right)$. Moreover, the the local energy estimate method from [3] is developed till now for radial potentials $a(|x|)$ only. These reasons lead us to construct some new variant of semiclassical method. On the contrary with [1], we consider a family of first eigenvalues of non-linear Schrödinger operator directly connected with equation (1.1), instead of eigenvalues $\mu_{i}$ (1.10) of auxiliary linear Schrödinger operator. As a consequence, we do not need regularizing effects for solutions of problem (1.1)(1.3). But this means also that we can not use Lieb-Thirring formula [16] to estimate the first eigenvalue. Therefore, we provide estimations of eigenvalues thanks to suitable Sobolev embedding inequalities. 
Thus let us denote for arbitrary potential $a(x) \geqslant 0$ the function

(1.14) $M_{a}(s):=\operatorname{meas}\{x \in \Omega: a(x) \leq s\}$.

Then main assumptions on the degeneration of $a(x)$ are:

(1.15) $\int_{0}^{1} s^{-1} M_{a}(s)^{\theta} d s<+\infty, \theta=\min \left(\frac{2 m}{N}, 1\right), N \neq 2 m$,

and

(1.16) $\int_{0}^{1} s^{-1} M_{a}(s)\left(-\ln M_{a}(s)\right) d s<+\infty$ for $N=2 m$.

For a function $a(x)$ satisfying (1.15) or (1.16) the set where it takes small values is small enough. For instance, if $a(x) \geq \gamma>0$ then $M_{a}(s)=0 \forall s<\gamma$ and, as consequence, integrals are finite. On the contrary, if $a(x)=0$ on a set of positive measure, integrals becomes infinite.

Definition 1.2 A function $u$ belonging to the space $\mathcal{C}\left([0, \infty), L^{2}(\Omega)\right) \bigcap L_{\text {loc }}^{2}([0, \infty)$, $\left.W_{0}^{m, 2}(\Omega)\right)$ is a weak solution of problem (1.1)-(1.3) if initial condition (1.3) holds and if for any $\zeta \in L_{\text {loc }}^{2}\left([0,+\infty), W_{0}^{m, 2}(\Omega)\right)$, there holds

$$
\left.\left.\int_{0}^{T} .1\right\rangle\left\langle u_{t}(t, .), \zeta(t, .)\right\rangle+\int_{\Omega}\left(\sum_{|\alpha|=m} a_{\alpha}\left(x, u, \ldots, D_{x}^{m} u\right) D_{x}^{\alpha} \zeta+a(x)|u|^{q-1} u \zeta\right) d x\right] d t=0 \text {, }
$$

for all $T>0$, where $\langle.,$.$\rangle is the paring of elements from \left(W_{0}^{m, 2}(\Omega)\right)^{*}$ and $W_{0}^{m, 2}(\Omega)$, $W_{0}^{m, 2}(\Omega)$ being the closure in the norm $W^{m, 2}(\Omega)$ of the space $\mathcal{C}_{0}^{m}(\Omega)$.

Main results are the following :

Theorem 1.1 Under assumptions (1.4) and (1.7),

a) if $N \neq 2 m$ and (1.15) holds, then all weak solutions of problem (1.1)-(1.3) have the EFT-property,

b) if $N=2 m$ and (1.16) holds, then all solutions of problem (1.1)-(1.3) have the EFT-property.

Remark 1.3 Condition (1.15) by $m=1, N>2$ in the case of radial potential $a(|x|)$ implies condition (1.13).

Additionally, for second order equation $(m=1)$, we improved the KV-method for the Dirichlet problem and the results holds as follows:

Theorem 1.2 Let $m=1$ in equation (1.1) and

(1.18) $\int_{0}^{1} s^{-1} M_{a}(s)^{\frac{2}{N}} d s<+\infty$,

then all solutions of problem (1.1)-(1.3) have the EFT-property. 


\section{Proof of Theorem 1.1}

The general principle is to find a lower bound for the function $U(t):=\int_{\Omega}\left(\left|D_{x}^{m} u\right|^{2}+a(x)|u|^{1+q}\right) d x$, with the help of the function

(2.1) $\lambda_{1}(h)=\inf \left\{\int_{\Omega}\left(\left|D_{x}^{m} v\right|^{2}+a(x)|v|^{1+q}\right) d x, v \in W_{0}^{m, 2}(\Omega),\|v\|_{L^{2}(\Omega)}^{2}=h\right\}$

The key-stone of this section is the following :

\section{Proposition 2.1 If}

$$
\int_{0}^{1} \frac{1}{\lambda_{1}(h)} d h<+\infty
$$

then all solutions of problem (1.1)-(1.3) vanish in a finite time and in this case,

(2.3) $T \leq \frac{1}{2} \int_{0}^{\left\|u_{0}\right\|_{L^{2}(\Omega)}^{2}} \frac{1}{\lambda_{1}(h)} d h$.

Proof: Using $\zeta=u$ in (1.17) gives for all $0 \leq s<t$,

$$
\int_{s}^{t}\left[\left\langle u_{\tau}(\tau, .), u(\tau, .)\right\rangle+\int_{\Omega}\left(\sum_{|\alpha|=m} a_{\alpha}\left(x, u, \ldots, D_{x}^{m} u\right) D_{x}^{\alpha} u(x, \tau)+a(x)|u|^{q+1}\right) d x\right] d \tau=0,
$$

which implies by formula of integration by parts (see [20]),

$$
\frac{1}{2} \int_{\Omega}\left(|u(t, .)|^{2}-|u(0, .)|^{2}\right) d x+\int_{0}^{t} \int_{\Omega}\left(\sum_{|\alpha|=m} a_{\alpha}\left(x, u, \ldots, D_{x}^{m} u\right) D_{x}^{\alpha} u+a(x)|u|^{q+1}\right) d x d \tau=0 .
$$

But the second term is absolutely continuous with respect to time. Therefore the first term is also absolutely continous and has derivative a.e. with respect to time which leads to

$$
\frac{1}{2} \frac{d}{d t}\left(\|u\|_{L^{2}(\Omega)}^{2}\right)+\int_{\Omega}\left(\sum_{|\alpha|=m} a_{\alpha}\left(x, u, \ldots, D_{x}^{m} u\right) D_{x}^{\alpha} u+a(x)|u|^{q+1}\right) d x=0 .
$$

Clearly, from the property (1.6) and the definition (2.1) of $\lambda_{1}(h)$, a.e.,

$$
c \int_{\Omega}\left(\sum_{|\alpha|=m} a_{\alpha}\left(x, u, \ldots, D_{x}^{m} u\right) D_{x}^{\alpha} u+a(x)|u|^{q+1}\right) d x \geq \lambda_{1}\left(\|u(., t)\|_{L^{2}(\Omega)}^{2}\right),
$$


where $c=\max (C, 1), C$ from (1.6). As a consequence, a.e.,

$$
\frac{1}{2} \frac{d}{d t}\left(\|u(., t)\|_{L^{2}(\Omega)}^{2}\right)+c \lambda_{1}\left(\|u(., t)\|_{L^{2}(\Omega)}^{2}\right) \leq 0, \quad c>0 .
$$

We have an ordinary differential inequality for the function $y(t)=\|u(., t)\|_{L^{2}(\Omega)}^{2}$. Therefore the end of the proof is straightforward by solving of obtained differential inequality.

Now, from Proposition 2.1, we need an estimate for $\lambda_{1}(h)$ from below. For this purpose, rough estimates of $v_{h}$ in $L^{\infty}$-norm and $\lambda_{1}(h)$ by above are indispensable. But (1.15) (or (1.16)) does not give directly an a-priori estimate of $\lambda_{1}(h)$. It is why we use a trick. Suppose that $O$ belongs to $\Omega$. We define

$$
\widetilde{a}(x):=a(x) \exp \left(-\frac{1}{|x|^{\alpha}}\right), \alpha>0
$$

In a same way, $\widetilde{\lambda}_{1}(h)=\inf \left\{\int_{\Omega}\left(\left|D_{x}^{m} v\right|^{2}+\widetilde{a}(x)|v|^{1+q}\right) d x, v \in W_{0}^{m, 2}(\Omega),\|v\|_{L^{2}(\Omega)}^{2}=h\right\}$. Since $0 \leq \widetilde{a}(x) \leq a(x), \widetilde{\lambda}_{1}(h) \leq \lambda_{1}(h)$ for all $h>0$. Hence, $\int_{0}^{1} \frac{1}{\lambda_{1}(h)} d h \leq \int_{0}^{1} \frac{1}{\widetilde{\lambda}_{1}(h)} d h$. So, if $\int_{0}^{1} \frac{1}{\widetilde{\lambda}_{1}(h)} d h<+\infty$, we get a finite extinction time.

For $N \neq 2 m$, if $\alpha>0$ is small enough, $x \mapsto \exp \left(-\frac{1}{|x|^{\alpha}}\right)$ satisfies (1.15) by Proposition 4.2. By Theorem 4.1, both $a(x)$ and $\widetilde{a}(x)$ satisfy the same condition (1.15) but $\widetilde{a}(x)$ holds the a-priori estimate

$$
\widetilde{a}(x) \leq C \exp \left(-\frac{1}{|x|^{\alpha}}\right)
$$

For $N=2 m$, by Proposition 4.4, if $\alpha>0$ is small enough, $x \mapsto \exp \left(-\frac{1}{|x|^{\alpha}}\right)$ satisfies (1.16). In a same way, by Theorem 4.1 , both $a(x)$ and $\widetilde{a}(x)$ satisfy the same condition (1.16) but $\widetilde{a}(x)$ holds also estimate (2.5). With estimate (2.5), we get

Lemma 2.1 There exists some $C>0$ such that for $h>0$ small enough, (2.5) implies (2.6) $\widetilde{\lambda}_{1}(h) \leq C h(-\ln h)^{\frac{2 m}{\alpha}}$.

Proof: The proof is an adaptation of [13]. Let $v \in C_{0}^{\infty}(B)\left(B\right.$ is the unit-ball of $\mathbb{R}^{N}$ ) with $v \geq 0$ and $\|v\|_{L^{2}(\Omega)}=1$, so by homogeneity,

$$
\widetilde{\lambda}_{1}(h) \leq h \int_{\Omega}\left|D^{m} v\right|^{2} d x+h^{\frac{1+q}{2}} \int_{\Omega} \widetilde{a}(x)|v(x)|^{1+q} d x .
$$


Let $0<r \leq r_{0}$. We set $v_{r}(x)=v\left(\frac{x}{r}\right)$. Then,

$$
\int_{\Omega} v_{r}^{2}(x) d x=\int_{B_{r}} v_{r}^{2}(x) d x=\int_{B_{r}} v^{2}\left(\frac{x}{r}\right) d x=r^{N} \int_{B} v^{2}(y) d y=r^{N},
$$

with a translation. As a consequence, $\left\|\frac{v_{r}}{r^{\frac{N}{2}}}\right\|_{L^{2}(\Omega)}=1$. On the other hand, $D_{x}^{\alpha} v_{r}(x)=r^{-|\alpha|} D_{\xi}^{\alpha} v\left(\frac{x}{r}\right)$. As a consequence, there exists $C>0$ such that

$$
\int_{\Omega}\left|D_{x}^{m} v_{r}\right|^{2} d x=\int_{B_{r}}\left|D_{x}^{m} v_{r}\right|^{2} d x \leq r^{N} \frac{C}{r^{2 m}} .
$$

Then by using $\frac{v_{r}}{r^{\frac{N}{2}}}$ in the definition of $\widetilde{\lambda}_{1}(h)$,

$$
\widetilde{\lambda}_{1}(h) \leq C \frac{h}{r^{2 m}}+h^{\frac{1+q}{2}} \frac{r^{N}}{r^{\frac{(1+q)}{2}}} \int_{B} \widetilde{a}(r y)|v(y)|^{1+q} d y .
$$

If we estimate $\int_{B} \widetilde{a}(r y)|v(y)|^{1+q} d y$ by $C \exp \left(-\frac{1}{r^{\alpha}}\right) \int_{B}|v(y)|^{1+q} d y$, then

$$
\widetilde{\lambda}_{1}(h) \leq C^{\prime}\left(\frac{h}{r^{2 m}}+h^{\frac{1+q}{2}} r^{N \frac{(1-q)}{2}} \exp \left(-\frac{1}{r^{\alpha}}\right)\right) \text {. }
$$

To balance both terms, we choose $r=\frac{1}{(-\ln h)^{\frac{1}{\alpha}}}$. By substituting $r$,

$$
\widetilde{\lambda}_{1}(h) \leq C\left(h(-\ln h)^{\frac{2 m}{\alpha}}+h^{\frac{1+q}{2}+1} \frac{1}{(-\ln h)^{\frac{N(1-q)}{2 \alpha}}}\right) \leq C^{\prime} h(-\ln h)^{\frac{2 m}{\alpha}},
$$

for $h$ small enough which completes the proof.

We introduce the functional

(2.7) $\widetilde{F}(v)=\int_{\Omega}\left(\left|D^{m} v\right|^{2}+\widetilde{a}(x)|v|^{1+q}\right) d x$,

for all $v \in W_{0}^{m, 2}(\Omega)$. Hence, there exists for all $h>0$, a function $\widetilde{v_{h}} \in W_{0}^{m, 2}(\Omega)$ such that

(2.8) $\left\|\widetilde{v_{h}}\right\|_{L^{2}(\Omega)}^{2}=h$ and $\widetilde{\lambda}_{1}(h) \leq \widetilde{F}\left(\widetilde{v_{h}}\right) \leq 2 \widetilde{\lambda}_{1}(h)$,

since $\widetilde{\lambda}_{1}(h)>0$. We prove Theorem 1.1 by estimating $\widetilde{\lambda}_{1}(h)$ from below. First, we deal with $N \neq 2 m$. 
Proposition 2.2 Under assumptions (1.4), (1.7) and (1.15), for $N \neq 2 m$, there exist $C>0, C^{\prime}>0$ and $\eta>0$ such that for $h$ small enough,

(2.9) $C \leq \frac{\widetilde{\lambda}_{1}(h)}{h}\left[\operatorname{meas}\left\{C^{\prime} h^{\eta} \geq \widetilde{a}(x)\right\}\right]^{\theta}, \theta=\min \left(\frac{2 m}{N}, 1\right)$.

Proof: Let $v \in W_{0}^{m, 2}(\Omega)$ with $\|v\|_{L^{2}(\Omega)}>0$. From Definition (2.7) of functional $\widetilde{F}$, it follows

$$
\int_{\Omega}\left|D^{m} v\right|^{2} d x d x=\int_{\{x:|v|>0\}}|v|^{2} H(v, x) d x, H(v, x)=\frac{\widetilde{F}(v)}{\|v\|_{L^{2}(\Omega)}^{2}}-\frac{\widetilde{a}(x)}{|v|^{1-q}},
$$

which yields

$$
C_{1}\left\|D_{x}^{m} v\right\|_{L^{2}(\Omega)}^{2} \leq \int_{\{x:|v|>0\}}|v|^{2} H(v, x)^{+} d x, H(v, x)^{+}:=\max (0, H(v, x)) .
$$

Since $v \in W_{0}^{m, 2}(\Omega)$, from the Sobolev imbedding, it follows :

(2.12) $\|v\|_{L^{p^{*}(\Omega)}}^{2} \leq C_{3}\left\|D_{x}^{m} v\right\|_{L^{2}(\Omega)}^{2}$,

where constant $C_{3}$ does not depend on $v$ and $p^{*}$ is defined by

(2.13) $p^{*}=\left\{\begin{array}{lll}\frac{2 N}{N-2 m} & \text { if } \quad N>2 m \\ +\infty & \text { if } \quad N<2 m\end{array}\right.$.

Combining estimate (2.12) and equality (2.10) we obtain:

$$
C_{4}\|v\|_{L^{p^{*}}(\Omega)}^{2} \leq \int_{\{x:|v|>0\}}|v|^{2} H(v, x)^{+} d x
$$

Using Hölder's inequality for estimating term in right-hand side of last inequality, we obtain

$$
C_{4}\|v\|_{L^{p^{*}}(\Omega)}^{2} \leq\|v\|_{L^{p^{*}}(\Omega)}^{2}\left[\int_{\{x:|v|>0\}}\left(H(v, x)^{+}\right)^{\frac{p^{*}}{p^{*}-2}} d x\right]^{\frac{p^{*}-2}{p^{*}}},
$$

where $\frac{p^{*}}{p^{*}-2}=\frac{p^{*}-2}{p^{*}}=1$ if $p^{*}=+\infty$. This last inequality yields to

$$
0<C_{4} \leq\left[\int_{\{x:|v|>0\}}\left(H(v, x)^{+}\right)^{\frac{p^{*}}{p^{*}-2}} d x\right]^{\frac{p^{*}-2}{p^{*}}} .
$$

where $H$ is from (2.10). From this estimate follows easily

$$
0<C_{4} \frac{\widetilde{F}(v)}{\|v\|_{L^{2}(\Omega)}^{2}}[\operatorname{meas}(\{x:|v|>0\} \bigcap\{H(v, x) \geq 0\})]^{\frac{p^{*}-2}{p^{*}}} .
$$


As a consequence, we obtain

$$
\begin{aligned}
& C_{4} \leq \frac{\widetilde{F}(v)}{\|v\|_{L^{2}(\Omega)}^{2}}[\operatorname{meas}(\{x:|v|>0\} \bigcap\{H(v, x) \geq 0\})]^{\frac{2 m}{N}}, N-2 m>0, \\
& C_{4} \leq \frac{\widetilde{F}(v)}{\|v\|_{L^{2}(\Omega)}^{2}}[\operatorname{meas}(\{x:|v|>0\} \bigcap\{H(v, x) \geq 0\})], N-2 m<0 .
\end{aligned}
$$

Therefore, for $v=\widetilde{v_{h}}$,

$$
C_{4} \leq \frac{2 \widetilde{\lambda}_{1}(h)}{h}[\operatorname{meas}\{x: \Gamma(h, x) \geq 0\}]^{\frac{2 m}{N}}, N-2 m>0,
$$

where $\Gamma(h, x)=\frac{2 \widetilde{\lambda}_{1}(h)}{h}\left|\widetilde{v_{h}}\right|^{1-q}-\widetilde{a}(x)$,

$$
C_{4} \leq \frac{2 \widetilde{\lambda}_{1}(h)}{h}[\operatorname{meas}\{x: \Gamma(h, x) \geq 0\}], N-2 m<0 .
$$

Now, we have to estimate $\left|\widetilde{v}_{h}\right|^{1-q}$ from above. By definition, we know that $\left\|\widetilde{v}_{h}\right\|_{L^{2}}^{2}=h$ so for all $\varepsilon>0$,

$$
\int_{\Omega} \widetilde{v}_{h}^{2} d x \geq \int_{\left\{x: \widetilde{v}_{h}^{2} \geq \varepsilon\right\}} \widetilde{v}_{h}^{2} d x \geq \varepsilon \operatorname{meas}\left\{x: \widetilde{v}_{h}^{2} \geq \varepsilon\right\} .
$$

By setting $\varepsilon=h^{\gamma}$ with $0<\gamma<1$, we get

$(2.16) h^{1-\gamma} \geq \operatorname{meas}\left\{x: \widetilde{v}_{h}^{2} \geq h^{\gamma}\right\}=\operatorname{meas}\{x: R(h, x) \geq 0\}, R(h, x)=\left|\widetilde{v}_{h}\right|^{1-q}-h^{\frac{\gamma(1-q)}{2}}$.

With this inequality, it follows

$$
\begin{aligned}
& \text { meas }\{x: \Gamma(h, x) \geq 0\}=\operatorname{meas}(\{x: \Gamma(h, x) \geq 0\} \bigcap\{x: R(h, x) \geq 0\}) \\
& +\operatorname{meas}(\{x: \Gamma(h, x) \geq 0\} \bigcap\{x: R(h, x)<0\}) .
\end{aligned}
$$

But, on one hand,

$$
\text { meas }(\{x: \Gamma(h, x) \geq 0\} \bigcap\{x: R(h, x) \geq 0\}) \leq \operatorname{meas}\{x: R(h, x) \geq 0\} \leq h^{1-\gamma},
$$

and on the other hand,

$$
\text { meas }(\{x: \Gamma(h, x) \geq 0\} \bigcap\{x: R(h, x)<0\}) \leq \operatorname{meas}\left\{x: \frac{2 \widetilde{\lambda}_{1}(h)}{h} h^{\frac{\gamma(1-q)}{2}} \geq \widetilde{a}(x)\right\} .
$$

As a consequence, we have

(2.17) meas $\{x: \Gamma(h, x) \geq 0\} \leq h^{1-\gamma}+$ meas $\left\{x: \frac{2 \widetilde{\lambda}_{1}(h)}{h} h^{\frac{\gamma(1-q)}{2}} \geq \widetilde{a}(x)\right\}$. 
Hence,

$$
C_{4} \leq\left(\frac{2 \widetilde{\lambda}_{1}(h)}{h}\right)^{\frac{N}{2 m}}\left[h^{1-\gamma}+\operatorname{meas}\left\{x: \frac{2 \widetilde{\lambda}_{1}(h)}{h} h^{\frac{\gamma(1-q)}{2}} \geq \widetilde{a}(x)\right\}\right], N-2 m>0 .
$$

From (2.6), $\frac{\widetilde{\lambda}_{1}(h)}{h} \leq C(-\ln h)^{\frac{2 m}{\alpha}}$ which yields for $h$ small enough,

$$
\left(\frac{2 \widetilde{\lambda}_{1}(h)}{h}\right)^{\frac{N}{2 m}} h^{1-\gamma} \leq 3 C h^{1-\gamma}(-\ln h)^{\frac{N}{\alpha}} \rightarrow 0
$$

when $h \rightarrow 0$. So there exists $C_{5}>0$ such that for $h$ small enough,

$$
C_{5} \leq\left(\frac{2 \widetilde{\lambda}_{1}(h)}{h}\right)^{\frac{N}{2 m}} \operatorname{meas}\left\{x: \frac{2 \widetilde{\lambda}_{1}(h)}{h} h^{\frac{\gamma(1-q)}{2}} \geq \widetilde{a}(x)\right\}, N-2 m>0 .
$$

Since $\gamma>0$, there exists $C^{\prime}>0$ and $\eta>0$ such that for $h$ small enough, $\frac{2 \widetilde{\lambda}_{1}(h)}{h} h^{\frac{\gamma(1-q)}{2}} \leq C^{\prime} h^{\eta}$. Consequently,

(2.18) $C_{5} \leq\left(\frac{2 \widetilde{\lambda}_{1}(h)}{h}\right)^{\frac{N}{2 m}} \operatorname{meas}\left\{x: C^{\prime} h^{\eta} \geq \widetilde{a}(x)\right\}$.

If $N-2 m<0$, we have in a very similar way,

(2.19) $C_{5} \leq \frac{2 \widetilde{\lambda}_{1}(h)}{h} \operatorname{meas}\left\{x: C^{\prime} h^{\eta} \geq \widetilde{a}(x)\right\}$,

which leads to the conclusion.

Proof of Theorem 1.1 for $N \neq 2 m$. Clearly, from (2.9),

$$
\int_{0}^{1} \frac{d h}{\widetilde{\lambda}_{1}(h)} \leq \int_{0}^{1} \frac{\operatorname{meas}\left\{x: C^{\prime} h^{\eta} \geq \widetilde{a}(x)\right\}^{\theta}}{h} d h,
$$

where $\theta$ is from (1.15). If we set $s=C^{\prime} h^{\eta}, \frac{d s}{s}=\eta \frac{d h}{h}$ and so

$$
\int_{0}^{1} \frac{d h}{\widetilde{\lambda}_{1}(h)} \leq \frac{1}{\eta} \int_{0}^{C^{\prime}} \frac{\text { meas }\{x: s \geq \widetilde{a}(x)\}^{\theta}}{s} d s
$$

Since

$$
\int_{0}^{1} \frac{d h}{\lambda_{1}(h)} \leq \int_{0}^{1} \frac{d h}{\widetilde{\lambda}_{1}(h)}
$$

we get the conclusion thank to Proposition 2.1. 
Proposition 2.3 Under assumptions (1.4), (1.7) and (1.16), for $N=2 m$, there exists $C>0$ such that for $h$ small enough,

$$
C \leq \frac{\widetilde{\lambda}_{1}(h)}{h}\left(\widehat{B}^{-1}\left(\operatorname{meas}\left\{x:\left|\widetilde{v}_{h}\right|^{1-q} \frac{2 \widetilde{\lambda}_{1}(h)}{h} \geq \widetilde{a}(x)\right\}\right)^{-1}\right)^{-1}
$$

where $\widehat{B}(s)=(s+1) \ln (s+1)-s$ is the complementary function of $B(t)=e^{t}-1-t$ in the sense of Orlicz space (see [23]).

Proof: Let $v \in W_{0}^{m, 2}(\Omega)$ with $\|v\|_{L^{2}(\Omega)}>0$. We return to the functional $\widetilde{F}$ from (2.7) again. Let $\tilde{v}_{h} \in W_{0}^{m, 2}(\Omega)$ is from (2.8). Due to optimal imbedding (see [27]) the following estimate holds:

$(2.21)\left\|\widetilde{v}_{h}\right\|_{L_{A}(\Omega)} \leq C_{3}\left\|D_{x}^{m} \widetilde{v}_{h}\right\|_{L^{2}(\Omega)}^{2}$,

where $L_{A}(\Omega)$ is the Orlicz space related to $A(t)=\exp \left(t^{\frac{p}{p-1}}\right)$ (see [23]) and $C_{3}$ is a positive constant which does not depend on $\widetilde{v}_{h}$. Thus, we deduce from (2.21) and (2.11) for $v=\widetilde{v}_{h}$ :

$$
C_{4}\left\|\widetilde{v}_{h}\right\|_{L_{A}(\Omega)}^{2} \leq \int_{\left\{x:\left|\widetilde{v}_{h}\right|>0\right\}}\left|\widetilde{v}_{h}\right|^{2}\left(\frac{\Gamma(h, x)}{\left|\widetilde{v}_{h}\right|^{1-q}}\right) d x,
$$

where $\Gamma(h, x)$ is from $(2.15)$. So,

$$
C_{4}\left\|\widetilde{v}_{h}\right\|_{L_{A}(\Omega)}^{2} \leq \int_{\left\{x:\left|\widetilde{v}_{h}\right|>0\right\}}\left|\widetilde{v}_{h}\right|^{2}\left(\frac{\Gamma(h, x)}{\left|\widetilde{v}_{h}\right|^{1-q}}\right)^{+} d x .
$$

By setting $B(t)=e^{t}-1-t$ and using the generalized version of Hölder's inequality (4.1),

$$
C_{4}\left\|\widetilde{v}_{h}\right\|_{L_{A}(\Omega)}^{2} \leq\left\|\widetilde{v}_{h}^{2}\right\|_{L_{B}\left(\left\{x:\left|\widetilde{v}_{h}\right|>0\right\}\right)}\left\|\left(\frac{\Gamma(h, x)}{\left|\widetilde{v}_{h}\right|^{1-q}}\right)^{+}\right\|_{L_{\widehat{B}\left(\left\{x:\left|\widetilde{v}_{h}\right|>0\right\}\right)}} .
$$

By Proposition 4.5, $\left\|\widetilde{v}_{h}\right\|_{L_{A}(\Omega)}^{2}=\left\|\widetilde{v}_{h}^{2}\right\|_{L_{M}(\Omega)}$. But $B(t)=e^{t}-1-t \leq e^{t}-1=A(\sqrt{t})=$ $M(t)$ implies by Proposition 4.6, $\left\|\widetilde{v}_{h}^{2}\right\|_{L_{B}(\Omega)} \leq\left\|\widetilde{v}_{h}^{2}\right\|_{L_{M}(\Omega)}$ and so,

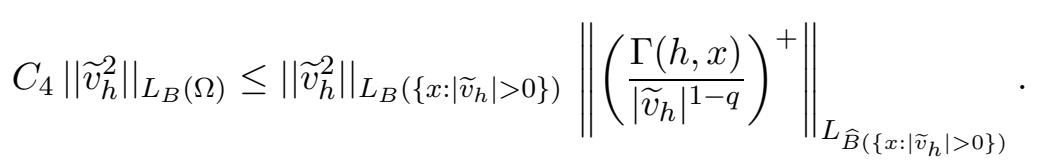

Furthermore, $\left\|\widetilde{v}_{h}^{2}\right\|_{L_{B}\left(\left\{x:\left|\widetilde{v}_{h}\right|>0\right\}\right)} \leq\left\|\widetilde{v}_{h}^{2}\right\|_{L_{B}(\Omega)}$ and as a consequence,

$$
C_{4} \leq\left\|\left(\frac{\Gamma(h, x)}{\left|\widetilde{v}_{h}\right| 1-q}\right)^{+}\right\|_{L_{\widehat{B}\left(\left\{x:\left|\widetilde{v}_{h}\right|>0\right\}\right)}} .
$$


We have

$$
\begin{aligned}
& \left\|\left(\frac{\Gamma(h, x)}{\left|\widetilde{v}_{h}\right|^{1-q}}\right)^{+}\right\|_{L_{\widehat{B}\left(\left\{x:\left|\widetilde{v}_{h}\right|>0\right\}\right)}}=\left\|\left(\frac{\Gamma(h, x)}{\left|\widetilde{v}_{h}\right|^{1-q}}\right)^{+}\right\|_{L_{\widehat{B}\left(\left\{x: \widetilde{v}_{h} \mid>0\right\} \cap\{x: \Gamma(h, x) \geq 0\}\right)}} \\
& \leq\left\|\left(\frac{\Gamma(h, x)}{\left|\widetilde{v}_{h}\right|^{1-q}}\right)^{+}\right\|_{L_{\widehat{B}(\{x: \Gamma(h, x) \geq 0\})}},
\end{aligned}
$$

by Proposition 4.7. With Proposition 4.8, we get

$$
C_{4} \leq\left\|\left(\frac{\Gamma(h, x)}{\left|\widetilde{v}_{h}\right|^{1-q}}\right)^{+}\right\|_{L^{\infty}(E)}\left(\widehat{B}^{-1}\left((\operatorname{meas}\{x: \Gamma(h, x) \geq 0\})^{-1}\right)\right)^{-1} .
$$

We have

$$
\left\|\left(\frac{\Gamma(h, x)}{\left|\widetilde{v}_{h}\right|^{1-q}}\right)^{+}\right\|_{L^{\infty}(E)} \leq \frac{\widetilde{\lambda}_{1}(h)}{h},
$$

so

$$
C_{4} \leq \frac{2 \widetilde{\lambda}_{1}(h)}{h}\left(\widehat{B}^{-1}\left((\operatorname{meas}\{x: \Gamma(h, x) \geq 0\})^{-1}\right)\right)^{-1},
$$

when $h$ is small enough which leads to the conclusion.

Proof of Theorem 1.1 for $N=2 m$. If $b$ is the derivative of $B$ then $b^{-1}(s)=\ln (s+1)$ and

$$
\widehat{B}(s)=\int_{0}^{s} b^{-1}(\sigma) d \sigma \sim s \ln s,
$$

when $s \rightarrow+\infty$. So there exists $C_{0}>0$ such that for $s$ large enough, $\widehat{B}(s) \leq C_{0} s \ln s=D(s)$. Hence by Proposition $4.9, \widehat{B}^{-1}(s) \geq D^{-1}(s)$ always for $s$ large enough. Moreover, estimate $\ln D(s)=\ln C_{0}+\ln s+\ln (\ln s) \sim \ln s$ gives $s \sim \frac{D(s)}{C_{0} \ln D(s)}$, i.e., $D^{-1}(s) \sim \frac{s}{C_{0} \ln s}$. So there exists some positive $K$ such that for $s$ large enough, $\widehat{B}^{-1}(s) \geq D^{-1}(s) \geq K \frac{s}{\ln s}$. From (2.20), for $h$ small enough,

$$
C \leq \frac{\widetilde{\lambda}_{1}(h)}{h}(-\ln \text { meas }\{x: \Gamma(h, x) \geq 0\})(\text { meas }\{x: \Gamma(h, x) \geq 0\}) .
$$

If $0<\gamma<1$ then estimate $(2.17)$ is true, i.e.,

$$
\text { meas }\{x: \Gamma(h, x) \geq 0\} \leq h^{1-\gamma}+\text { meas }\left\{x: \frac{2 \widetilde{\lambda}_{1}(h)}{h} h^{\frac{\gamma(1-q)}{2}} \geq \widetilde{a}(x)\right\},
$$


which implies together with estimate (2.6) that meas $\{x: \Gamma(h, x) \geq 0\} \rightarrow 0$ when $h \rightarrow 0$. If for all positive $s$, we set

(2.22) $E(s)=s(-\ln s)$,

then $C \leq \frac{\widetilde{\lambda}_{1}(h)}{h} E($ meas $\{x: \Gamma(h, x) \geq 0\})$.

The function $E$ is increasing in a neighbourhood of zero so,

$$
E^{-1}\left(\frac{C h}{\widetilde{\lambda}_{1}(h)}\right) \leq \operatorname{meas}\{x: \Gamma(h, x) \geq 0\} .
$$

By (2.17),

$$
E^{-1}\left(\frac{C h}{\widetilde{\lambda}_{1}(h)}\right) \leq h^{1-\gamma}+\operatorname{meas}\left\{x: \frac{2 \widetilde{\lambda}_{1}(h)}{h} h^{\frac{\gamma(1-q)}{2}} \geq \widetilde{a}(x)\right\},
$$

i.e.,

$$
1 \leq \frac{h^{1-\gamma}}{E^{-1}\left(C h\left(\widetilde{\lambda}_{1}(h)\right)^{-1}\right)}+\frac{\operatorname{meas}\left\{x: \frac{2 \widetilde{\lambda}_{1}(h)}{h} h^{\frac{\gamma(1-q)}{2}} \geq \widetilde{a}(x)\right\}}{E^{-1}\left(C h\left(\widetilde{\lambda}_{1}(h)\right)^{-1}\right)} .
$$

But from (2.6),

$$
\frac{h^{1-\gamma}}{E^{-1}\left(C h\left(\widetilde{\lambda}_{1}(h)\right)^{-1}\right)} \leq \frac{h^{1-\gamma}}{E^{-1}\left(C^{\prime \prime}(-\ln h)^{\frac{-2 m}{\alpha}}\right)} \rightarrow 0
$$

since when $s \rightarrow 0, E^{-1}(s) \sim \frac{s}{-\ln s}$. Consequently, for $h$ small enough,

$$
E^{-1}\left(C h\left(\widetilde{\lambda}_{1}(h)\right)^{-1}\right) \leq 2 \text { meas }\left\{x: \frac{2 \widetilde{\lambda}_{1}(h)}{h} h^{\frac{\gamma(1-q)}{2}} \geq \widetilde{a}(x)\right\} .
$$

Always from (2.6), there exist $C^{\prime}>0$ and $\eta>0$ such that,

$$
\text { meas }\left\{x: \frac{2 \widetilde{\lambda}_{1}(h)}{h} h^{\frac{\gamma(1-q)}{2}} \geq \widetilde{a}(x)\right\} \leq \text { meas }\left\{x: C^{\prime} h^{\eta} \geq \widetilde{a}(x)\right\},
$$

which gives

$$
C \leq \frac{\widetilde{\lambda}_{1}(h)}{h} E\left(2 \text { meas }\left\{x: C^{\prime} h^{\eta} \geq \widetilde{a}(x)\right\}\right) .
$$


We easily deduce that there exist some $K>0$ and $\delta>0$ such that

$$
K \int_{0}^{\delta} \frac{d h}{\widetilde{\lambda}_{1}(h)} \leq \int_{0}^{\delta}\left(\operatorname{meas}\left\{x: C^{\prime} h^{\eta} \geq \widetilde{a}(x)\right\}\right)\left(-\ln \left(\operatorname{meas}\left\{x: C^{\prime} h^{\eta} \geq \widetilde{a}(x)\right\}\right)\right) \frac{d h}{h} .
$$

If we set $s=C^{\prime} h^{\eta}, \frac{d s}{s}=\eta \frac{d h}{h}$ and so

$$
K \int_{0}^{\delta} \frac{d h}{\widetilde{\lambda}_{1}(h)} \leq \frac{1}{\eta} \int_{0}^{\delta C^{\prime}}(\text { meas }\{x: s \geq \widetilde{a}(x)\})(-\ln (\operatorname{meas}\{x: s \geq \widetilde{a}(x)\})) \frac{d s}{s} .
$$

Since $\int_{0}^{1} \frac{d h}{\lambda_{1}(h)} \leq \int_{0}^{1} \frac{d h}{\widetilde{\lambda}_{1}(h)}$, we get the conclusion thank to Proposition 2.1.

We can derive some useful corollaries.

Corollary 2.1 Let $f:(0,+\infty) \rightarrow(0,+\infty)$ be a continuous nonincreasing function such that $f(a(x)) \in L^{1}(\Omega)$ and $\int_{0}^{1} s^{-1} f(s)^{-\theta} d s<+\infty$ where $\theta$ is defined in (1.15). Then, under assumptions (1.4) and (1.7), for $N \neq 2 m$, all solutions of problem (1.1)(1.3) vanish in a finite time.

Proof: If $s>0$, meas $\{x: a(x) \leq s\}=$ meas $\{x: f(a(x)) \geq f(s)\}$ and so,

$$
\text { meas }\{x: a(x) \leq s\} \leq f(s)^{-1} \int_{\Omega} f(a(x)) d x,
$$

and we conclude with Theorem 1.1.

Corollary 2.2 Let $f:(0,+\infty) \rightarrow(0,+\infty)$ be a continuous nonincreasing function such that $f(a(x)) \in L^{1}(\Omega)$ and

$$
\int_{0}^{1} s^{-1} f(s)^{-1} \ln f(s) d s<+\infty
$$

Then, under assumptions (1.4) and 1.7, for $N=2 m$, all solutions of problem (1.1)(1.3) vanish in a finite time.

Proof: The function $f$ has a limit when $t$ tends to zero. By (2.23), this limit is $+\infty$. If $s>0$ is small enough, as in the previous proof,

$$
\text { meas }\{x: a(x) \leq s\} \leq f(s)^{-1} \int_{\Omega} f(a(x)) d x .
$$

We set $E(s)=s(-\ln s)$ for all positive $s$ and since $E$ is an increasing function in a neighbourhood of zero, there exists some $\delta>0$ such that

$$
\int_{0}^{\delta} s^{-1} E\left(M_{a}(s)\right) d s \leq \int_{0}^{\delta} s^{-1} E\left(f(s)^{-1} \int_{\Omega} f(a(x)) d x\right) d s
$$


which leads to

$\int_{0}^{\delta} s^{-1} E\left(M_{a}(s)\right) d s \leq\left(\int_{\Omega} f(a(x)) d x\right) \int_{0}^{\delta} s^{-1} f(s)^{-1}\left(\ln f(s)-\ln \left(\int_{\Omega} f(a(x)) d x\right)\right) d s$.

But, as $f(s) \rightarrow+\infty$ when $s \rightarrow 0$, there exists some $C>0$ such that,

$$
\int_{0}^{\delta} s^{-1} E\left(M_{a}(s)\right) d s \leq C \int_{0}^{\delta} s^{-1} f(s)^{-1} \ln f(s) d s,
$$

and we conclude with Theorem 1.1.

There is a balance between both assumptions, i.e., $f$ has to get the right behaviour. For instance, in [2], they prove that for $m=1$,

$$
\ln \frac{1}{a} \in L^{p}(\Omega)
$$

with $p>\frac{N}{2}$ implies the extinction in a finite time for the Laplacian. From the previous corollary, for more general operators,

Corollary 2.3 Under assumptions (1.4), (1.7) and (2.24) for $N \neq 2 m$ and $p>\theta$, all solutions of problem (1.1)-(1.3) vanish in a finite time.

We can also find a Dini-like condition in the radial case in the spirit of [3].

Corollary 2.4 Assume that $a(x)=\exp \left(-\frac{\omega(|x|)}{|x|^{N \theta}}\right)$ with $\omega$ a non decreasing and nonnegative function on $(0,1]$ and $\omega(s) \leq \omega_{0}, \forall s \in[0,1]$. If $\omega$ satisfies

$$
\int_{0}^{1} s^{-1} \omega(s) d s<+\infty
$$

under assumptions (1.4) and (1.8), for $N \neq 2 m$, one have a finite extinction time for all solutions of problem (1.1)-(1.3).

Proof: For $s>0, \operatorname{meas}\{x: a(x) \leq s\}=$ meas $\left\{x: \frac{\omega(|x|)}{|x|^{N \theta}} \geq-\ln s\right\}$. We take $x$ such that $\frac{\omega(|x|)}{|x|^{N \theta}} \geq-\ln s$. Since $\omega$ is bounded, $x$ satisfies $\frac{\omega_{0}}{|x|^{N \theta}} \geq-\ln s$ which leads to $|x| \leq\left(\frac{\omega_{0}}{-\ln s}\right)^{\frac{1}{N \theta}}$. By monotonicity of $\omega$, $\omega(|x|) \leq \omega\left(\left(\frac{\omega_{0}}{-\ln s}\right)^{\frac{1}{N \theta}}\right)$. Hence,

$$
\operatorname{meas}\{x: a(x) \leq s\} \leq \operatorname{meas}\left\{x:|x|^{N \theta} \leq \omega\left(\left(\frac{\omega_{0}}{-\ln s}\right)^{\frac{1}{N \theta}}\right)(-\ln s)^{-1}\right\} \text {. }
$$


But

meas $\left\{x:|x|^{N \theta} \leq \omega\left(\left(\frac{\omega_{0}}{-\ln s}\right)^{\frac{1}{N \theta}}\right)(-\ln s)^{-1}\right\}=C_{N}\left(\omega\left(\left(\frac{\omega_{0}}{-\ln s}\right)^{\frac{1}{N \theta}}\right)(-\ln s)^{-1}\right)^{\frac{1}{\theta}}$.

So,

$$
\operatorname{meas}\{x: a(x) \leq s\}^{\theta} \leq C_{N}^{\theta} \omega\left(\left(\frac{\omega_{0}}{-\ln s}\right)^{\frac{1}{\min (2 m, N)}}\right)(-\ln s)^{-1},
$$

which yields

$$
\int_{0}^{\frac{1}{e}} s^{-1} M_{a}(s)^{\theta} d s \leq C_{N}^{\theta} \int_{0}^{\frac{1}{e}} s^{-1}(-\ln s)^{-1} \omega\left(\left(\frac{\omega_{0}}{-\ln s}\right)^{\frac{1}{N \theta}}\right) d s .
$$

By the change of variable $\tau=\omega_{0}(-\ln s)^{-1}$, that is, $\tau^{-1} d \tau=(-\ln s)^{-1} s^{-1} d s$,

$$
\int_{0}^{\frac{1}{e}} s^{-1} M_{a}(s)^{\theta} d s \leq C_{N}^{\theta} \int_{0}^{\omega_{0}} \omega\left(\tau^{\frac{1}{N \theta}}\right) \tau^{-1} d \tau
$$

By the last change of variable $s=\tau^{\frac{1}{N \theta}}$, that is, $s^{-1} d s=\frac{1}{N \theta} \tau^{-1} d \tau$,

$$
\int_{0}^{\frac{1}{e}} s^{-1} M_{a}(s)^{\theta} d s \leq N \theta C_{N}^{\theta} \int_{0}^{\omega_{0}^{\frac{1}{N \theta}}} s^{-1} \omega(s) d s .
$$

Theorem 1.1 completes the proof.

Corollary 2.5 Assume that $a(x)=\exp \left(-\frac{\omega(|x|)}{|x|^{N}}\right)$ with $\omega$ a nondecreasing and nonnegative function on $(0,1]$ and $\omega(s) \leq \omega_{0}, \forall s \in[0,1]$. If $\omega$ satisfies

$$
\int_{0}^{1} s^{-1} \omega(s)(-\ln (\omega(s))-\ln s) d s<+\infty
$$

under assumptions (1.4) and (1.7), for $N=2 m$, one has a finite extinction time for all solutions of problem (1.1)-(1.3).

Proof: For $s>0, \operatorname{meas}\{x: a(x) \leq s\}=$ meas $\left\{x: \frac{\omega(|x|)}{|x|^{N}} \geq-\ln s\right\}$. We take $x$ such that $\frac{\omega(|x|)}{|x|^{N}} \geq-\ln s$. Since $\omega$ is bounded, $x$ satisfies $\frac{\omega_{0}}{|x|^{N}} \geq-\ln s$ which leads to $|x| \leq\left(\frac{\omega_{0}}{-\ln s}\right)^{\frac{1}{N}}$. By monotonicity of $\omega, \omega(|x|) \leq \omega\left(\left(\frac{\omega_{0}}{-\ln s}\right)^{\frac{1}{N}}\right)$. Hence,

$$
\operatorname{meas}\{x: a(x) \leq s\} \leq \operatorname{meas}\left\{x:|x|^{N} \leq \omega\left(\left(\frac{\omega_{0}}{-\ln s}\right)^{\frac{1}{N}}\right)(-\ln s)^{-1}\right\} \text {. }
$$


But meas $\left\{x:|x|^{N} \leq \omega\left(\left(\frac{w_{0}}{-\ln s}\right)^{\frac{1}{N}}\right)(-\ln s)^{-1}\right\}=C_{N} \omega\left(\left(\frac{\omega_{0}}{-\ln s}\right)^{\frac{1}{N}}\right)(-\ln s)^{-1}$. So, meas $\{x: a(x) \leq s\} \leq C_{N} \omega\left(\left(\frac{\omega_{0}}{-\ln s}\right)^{\frac{1}{N}}\right)(-\ln s)^{-1}$. If we set $E(s)=s(-\ln s)$ for all positive $s$, we get $E\left(M_{a}(s)\right) \leq E\left(C_{N} \omega\left(\left(\frac{\omega_{0}}{-\ln s}\right)^{\frac{1}{N}}\right)(-\ln s)^{-1}\right)$ for $s$ small enough since $E$ is an increasing function in a neighbourhood of zero. As a consequence, there exists some $\delta>0$ such that

$\int_{0}^{\delta} s^{-1} E\left(M_{a}(s)\right) d s \leq \int_{0}^{\delta} s^{-1} E\left(C_{N} \omega\left(\left(\frac{\omega_{0}}{-\ln s}\right)^{\frac{1}{N}}\right)(-\ln s)^{-1}\right) d s$,

which gives $\int_{0}^{\delta} s^{-1} E\left(M_{a}(s)\right) d s$

$\leq \int_{0}^{\delta} \frac{C_{N}}{s} \omega\left(\left(\frac{\omega_{0}}{-\ln s}\right)^{\frac{1}{N}}\right)(-\ln s)^{-1}\left(-\ln C_{N}-\ln \left(\omega\left(\left(\frac{\omega_{0}}{-\ln s}\right)^{\frac{1}{N}}\right)\right)+\ln (-\ln s)\right) d s$.

But $\omega$ satisfies (2.25) which means that by monotonicity, $\omega(s) \rightarrow 0$ when $s \rightarrow 0$. So for $s$ small enough, $-\ln C_{N}-\ln \left(\omega\left(\left(\frac{\omega_{0}}{-\ln s}\right)^{\frac{1}{N}}\right)\right)+\ln (-\ln s)$

$$
\leq 2\left(-\ln \left(\omega\left(\left(\frac{\omega_{0}}{-\ln s}\right)^{\frac{1}{N}}\right)\right)+\ln (-\ln s)\right) \text {. }
$$

Consequently, for some $0<\delta^{\prime}<\delta$, we get $\int_{0}^{\delta^{\prime}} s^{-1} E\left(M_{a}(s)\right) d s$

$$
\leq 2 C_{N} \int_{0}^{\delta^{\prime}} \omega\left(\left(\frac{\omega_{0}}{-\ln s}\right)^{\frac{1}{N}}\right)\left(-\ln \left(\omega\left(\left(\frac{\omega_{0}}{-\ln s}\right)^{\frac{1}{N}}\right)\right)+\ln (-\ln s)\right)(-s \ln s)^{-1} d s .
$$

By the change of variable $\tau=\omega_{0}(-\ln s)^{-1}$, that is, $\tau^{-1} d \tau=(-s \ln s)^{-1} d s$,

$$
\begin{aligned}
& \int_{0}^{\delta^{\prime}} s^{-1} E\left(M_{a}(s)\right) d s \\
& \quad \leq C_{N} \int_{0}^{\frac{\omega_{0}}{-\ln \delta^{\prime}}} \omega\left(\tau^{\frac{1}{N}}\right)\left(-\ln \left(\omega\left(\tau^{\frac{1}{N}}\right)\right)-\ln \tau+\ln \omega_{0}\right) \tau^{-1} d \tau .
\end{aligned}
$$

Hence, there exists $\delta^{\prime \prime}<\delta^{\prime}$ such that the following estimate holds :

$$
\int_{0}^{\delta^{\prime \prime}} s^{-1} E\left(M_{a}(s)\right) d s \leq 3 N C_{N} \int_{0}^{\frac{\omega_{0}}{-\ln \delta^{\prime \prime}} \omega} \omega\left(\tau^{\frac{1}{N}}\right)\left(-\ln \left(\omega\left(\tau^{\frac{1}{N}}\right)\right)-\ln \left(\tau^{\frac{1}{N}}\right)\right) \tau^{-1} d \tau .
$$


By the last change of variable $s=\tau^{\frac{1}{N}}$, that is, $s^{-1} d s=\frac{1}{N} \tau^{-1} d \tau$,

$$
\int_{0}^{\delta^{\prime \prime}} s^{-1} E\left(M_{a}(s)\right) d s \leq 3 N^{2} C_{N} \int_{0}^{\left(\frac{\omega_{0}}{-\ln \delta^{\prime \prime}}\right)^{\frac{1}{N}}} s^{-1} \omega(s)(-\ln (\omega(s))-\ln s) d s .
$$

This time also, Theorem 1.1 completes the proof.

\section{Second order case}

Here we prove Theorem 1.2. Our proof is a detailed analysis of sufficient condition of extinction of solutions obtained in [2] (see condition (4.2) in Theorem 4.2 from Appendix). They introduce the quantity

$$
\lambda_{1,2}(h)=\inf \left\{\int_{\Omega}\left(|\nabla v|^{2}+\frac{1}{h^{2}} a(x) v^{2}\right) d x: v \in W_{0}^{1,2}(\Omega), \int_{\Omega} v^{2} d x=1\right\}, h>0 .
$$

As in the previous section, for $\alpha>0$ small enough, changing function $a$ into

$$
\widetilde{a}(x)=a(x) \exp \left(-\frac{1}{|x|^{\alpha}}\right),
$$

does not change (1.18) but by defining in a very similar way

$$
\tilde{\lambda}_{1,2}(h)=\inf \left\{\int_{\Omega}\left(|\nabla v|^{2}+\frac{1}{h^{2}} \widetilde{a}(x) v^{2}\right) d x: v \in W_{0}^{1,2}(\Omega)\right\},
$$

we have the a-priori estimate by Corollary 2.23 in [13],

(3.2) $\widetilde{\lambda}_{1,2}(h) \leq C(-\ln h)^{\frac{2}{\alpha}}$.

Since $\widetilde{\lambda}_{1,2}(h) \leq \lambda_{1,2}(h)$ and $t \mapsto \frac{\ln t}{t}$ is a decreasing function for $t$ large enough, condition (4.2) from Theorem 4.2 (Appendix) is implied by

$$
\sum_{n=1}^{\infty} \frac{1}{\widetilde{\lambda}_{1,2}\left(\alpha_{n}^{\frac{1-q}{2}}\right)}\left(\ln \left(\widetilde{\lambda}_{1,2}\left(\alpha_{n}^{\frac{1-q}{2}}\right)\right)+\ln \left(\frac{\alpha_{n}}{\alpha_{n+1}}\right)+1\right)<+\infty
$$

As in [2], we transform condition (3.3) into a simpler form. The following theorem is an adaptation of Theorem 2.3 in [2].

Proposition 3.1 Condition (3.3) is equivalent to

$$
\int_{0}^{1} \frac{1}{h \widetilde{\lambda}_{1,2}(h)} d h<\infty
$$


Proof: By changing the sequence $\left\{\alpha_{n}\right\}$ into $\left\{\alpha_{n}^{\frac{2}{1-q}}\right\},(3.3)$ is equivalent to

$$
\sum_{n=1}^{\infty} \frac{1}{\widetilde{\lambda}_{1,2}\left(\alpha_{n}\right)}\left(\ln \left(\widetilde{\lambda}_{1,2}\left(\alpha_{n}\right)\right)+\ln \left(\frac{\alpha_{n}}{\alpha_{n+1}}\right)+1\right)<+\infty
$$

Suppose that (3.3) holds. Then, it implies that $\alpha_{n} \rightarrow 0$ and that $\widetilde{\lambda}_{1,2}\left(\alpha_{n}\right) \rightarrow \infty$ as $n$ tends to infinity. Clearly, $h \mapsto \widetilde{\lambda}_{1,2}(h)$ is a nonincreasing function which means that $\left\{\widetilde{\lambda}_{1,2}\left(\alpha_{n}\right)\right\}$ is a nondecreasing sequence. We use estimate of Theorem 2.3 in [2].

$$
\int_{\alpha_{n+1}}^{\alpha_{n}} \frac{1}{h \widetilde{\lambda}_{1,2}(h)} d h \leq \frac{1}{\widetilde{\lambda}_{1,2}\left(\alpha_{n}\right)} \ln \left(\frac{\alpha_{n}}{\alpha_{n+1}}\right), \forall n \geq 1,
$$

which yields

$$
\int_{0}^{\alpha_{1}} \frac{1}{h \widetilde{\lambda}_{1,2}(h)} d h \leq \sum_{n=1}^{\infty} \frac{1}{\widetilde{\lambda}_{1,2}\left(\alpha_{n}\right)} \ln \left(\frac{\alpha_{n}}{\alpha_{n+1}}\right)<+\infty .
$$

Conversely, suppose that (3.4) holds. We take the sequence $\alpha_{n}=n^{-n}$ as in [3]. Indeed, $\tilde{\lambda}_{1,2}\left(\alpha_{n}\right) \leq C(n \ln n)^{\frac{2}{\alpha}}$ leads to $\ln \left(\tilde{\lambda}_{1,2}\left(\alpha_{n}\right)\right) \leq C \ln n$ for $n$ large enough. Moreover,

(3.5) $\ln \left(\frac{\alpha_{n}}{\alpha_{n+1}}\right) \sim \ln n \Longrightarrow \ln \left(\widetilde{\lambda}_{1,2}\left(\alpha_{n}\right)\right) \leq C \ln \left(\frac{\alpha_{n}}{\alpha_{n+1}}\right)$,

always for $n$ large enough ( $C$ is a generic positive constant). Clearly, by monotonicity,

$$
\int_{\alpha_{n+1}}^{\alpha_{n}} \frac{1}{h \widetilde{\lambda}_{1,2}(h)} d h \geq \frac{1}{\widetilde{\lambda}_{1,2}\left(\alpha_{n+1}\right)} \ln \left(\frac{\alpha_{n}}{\alpha_{n+1}}\right), \forall n \geq 1 .
$$

Hence, thanks to (3.5), there exists $C>0$ such that for $n$ large enough,

$$
\int_{\alpha_{n+1}}^{\alpha_{n}} \frac{1}{h \widetilde{\lambda}_{1,2}(h)} d h \geq C \frac{1}{\widetilde{\lambda}_{1,2}\left(\alpha_{n+1}\right)} \ln \left(\frac{\alpha_{n+1}}{\alpha_{n+2}}\right) .
$$

So we get $\sum_{n=1}^{\infty} \frac{1}{\widetilde{\lambda}_{1,2}\left(\alpha_{n}\right)} \ln \left(\frac{\alpha_{n}}{\alpha_{n+1}}\right)<+\infty$. This implies (3.3).

Proof of Theorem 1.2. For $h$ small enough, we have the following estimate for $\widetilde{\lambda}_{1,2}(h)$ [2],

$$
0<C \leq \operatorname{meas}\left\{x \in \Omega: h^{-2} \widetilde{a}(x) \leq 3 \widetilde{\lambda}_{1,2}(h)\right\}\left(\widetilde{\lambda}_{1,2}(h)\right)^{\frac{N}{2}} .
$$

For this estimate, they use the Leib-Thirring formula about the counting number with some properties of semi-classical analysis [17]. By (3.2),

$$
0<C \leq \operatorname{meas}\left\{x \in \Omega: \widetilde{a}(x) \leq C h^{2}(-\ln h)^{\frac{2}{\alpha}}\right\}\left(\widetilde{\lambda}_{1,2}(h)\right)^{\frac{N}{2}} .
$$


So, for $h$ small enough, $C h^{2}(-\ln h)^{\frac{2}{\alpha}} \leq h$ which gives

$$
\frac{1}{\widetilde{\lambda}_{1,2}(h)} \leq C \operatorname{meas}\{x \in \Omega: \widetilde{a}(x) \leq h\}^{\frac{2}{N}} .
$$

As a consequence, for some $h_{0}>0$ small enough,

$$
\int_{0}^{h_{0}} \frac{1}{h \widetilde{\lambda}_{1,2}(h)} d h \leq C \int_{0}^{h_{0}} \frac{\operatorname{meas}\{x \in \Omega: \widetilde{a}(x) \leq h\}^{\frac{2}{N}}}{h} d h .
$$

We conclude with the following arguments :

$$
\int_{0}^{1} \frac{\operatorname{meas}\{x \in \Omega:|a(x)| \leq t\}^{\frac{2}{N}}}{t} d t<\infty
$$

implies by Theorem 4.1 and Proposition 4.2, $\int_{0}^{1} \frac{\operatorname{meas}\{x \in \Omega:|\widetilde{a}(x)| \leq t\}^{\frac{2}{N}}}{t} d t<\infty$ which yields $\int_{0}^{1} \frac{1}{h \widetilde{\lambda}_{1,2}(h)} d h<\infty$ and then by proposition 3.1 ,

$$
\sum_{n=1}^{\infty} \frac{1}{\widetilde{\lambda}_{1,2}\left(\alpha_{n}^{\frac{1-q}{2}}\right)}\left(\ln \left(\tilde{\lambda}_{1,2}\left(\alpha_{n}^{\frac{1-q}{2}}\right)\right)+\ln \left(\frac{\alpha_{n}}{\alpha_{n+1}}\right)+1\right)<\infty .
$$

This last inequality means that

$$
\sum_{n=1}^{\infty} \frac{1}{\lambda_{1,2}\left(\alpha_{n}^{\frac{1-q}{2}}\right)}\left(\ln \left(\lambda_{1,2}\left(\alpha_{n}^{\frac{1-q}{2}}\right)\right)+\ln \left(\frac{\alpha_{n}}{\alpha_{n+1}}\right)+1\right)<+\infty
$$

for some sequence $\left\{\alpha_{n}\right\}$. By Theorem 4.2 in Appendix, all solution vanish in a finite time.

Remark 3.1 If we assume that $a(x)$ is greater than a positive constant in a neighbourhood of the boundary of $\Omega$ then the related Neumann problem can be reduced to the former Dirichlet problem. Indeed, the solution of the Neumann problem vanishes in a finite time in a neighbourhood of the boundary of $\Omega$ and up to a shift in time, the solution satisfies the Dirichlet boundary condition.

\section{Appendix}

\subsection{The properties of classes $S_{\varphi}$}

Let $\varphi$ a function defined on $[0, \gamma]$ for some $\gamma>0$ which holds the following properties : 
1) $\varphi(0)=0$,

2) $\varphi$ is a nondecreasing function on $[0, \gamma]$,

3) $\varphi(t)>0, \forall t \in(0, \gamma]$,

4) there exist $C>0$ and $\gamma^{\prime} \in(0, \gamma]$ such that for all $\alpha, \beta$ in $\left[0, \gamma^{\prime}\right]$,

$$
\varphi(\alpha+\beta) \leq C(\varphi(\alpha)+\varphi(\beta)) .
$$

We set

$$
S_{\varphi}=\left\{a \in L^{\infty}(\Omega) \mid \exists c>0: \int_{0}^{c} \frac{\varphi(\operatorname{meas}\{x \in \Omega:|a(x)| \leq t\})}{t} d t<+\infty\right\} .
$$

We start with some basic properties.

\section{Proposition 4.1}

1. $a \in S_{\varphi} \Longleftrightarrow|a| \in S_{\varphi}$,

2. $1 \in S_{\varphi}$ (1 stands for the constant function equal to 1 on whole $\Omega$ ),

3. if $\psi$ satisfies (1), (2), (3) and $\varphi \leq \psi$ then $S_{\varphi} \supset S_{\psi}$,

4. $a \in S_{\varphi} \Longleftrightarrow \forall \lambda \in \mathbb{R}^{*}, \lambda a \in S_{\varphi}$.

5. $a \in S_{\varphi} \Longleftrightarrow \forall \kappa>0,|a|^{\kappa} \in S_{\varphi}$.

Proof: Let $a \in S_{\varphi}$ and $\lambda \in \mathbb{R}^{*}$. By the change of variable $t=|\lambda| \tau$,

$$
\int_{0}^{c} \frac{\varphi(\operatorname{meas}\{x \in \Omega:|\lambda a(x)| \leq t\})}{t} d t=\int_{0}^{\frac{c}{|\lambda|}} \frac{\varphi(\operatorname{meas}\{x \in \Omega:|a(x)| \leq \tau\})}{\tau} d \tau,
$$

which concludes the fourth assertion.

Let $a \in S_{\varphi}$ and $\kappa>0$. In a same way, by the change of variable $t=\tau^{\kappa}$,

$$
\int_{0}^{c} \frac{\varphi\left(\operatorname{meas}\left\{x \in \Omega:|a(x)|^{\kappa} \leq t\right\}\right)}{t} d t=\kappa \int_{0}^{c^{\frac{1}{\kappa}}} \frac{\varphi(\operatorname{meas}\{x \in \Omega:|a(x)| \leq \tau\})}{\tau} d \tau .
$$

The proof is complete.

Clearly, power functions satisfy (1), (2), (3) and (4).

Proposition 4.2 For $\alpha>0$ small enough, the function $w(x)=\exp \left(-\frac{1}{|x|^{\alpha}}\right)$ belongs to $S_{\varphi}$ where $\varphi(x)=x^{\beta}$ with $\beta>0$. 
Proof: By direct calculations,

$$
\varphi(\operatorname{meas}\{x \in \Omega:|w(x)| \leq t\})=\varphi\left(\operatorname{meas}\left\{x \in \Omega:|x|^{\alpha} \leq(-\ln t)\right\}\right)=C_{N} \frac{1}{(-\ln t)^{\frac{N \beta}{\alpha}}} .
$$

The main property of the set $S_{\varphi}$ is its stability with respect to the product.

Theorem 4.1 If $a$ and $b$ belong to $S_{\varphi}$ then $a b \in S_{\varphi}$.

Proof: The assumption $a, b \in S_{\varphi}$ implies that $a(x)>0$ and $b(x)>0$ a.e. on $\Omega$ so

$$
\lim _{t \rightarrow 0} \operatorname{meas}\left\{x \in \Omega:|b(x)|^{2} \leq t\right\}+\text { meas }\left\{x \in \Omega:|a(x)|^{2} \leq t\right\}=0 .
$$

Let $t>0$ small enough, i.e,

$$
\operatorname{meas}\left\{x \in \Omega:|b(x)|^{2} \leq t\right\}+\text { meas }\left\{x \in \Omega:|a(x)|^{2} \leq t\right\} \leq \gamma^{\prime} .
$$

Let us consider $\{x \in \Omega:|a(x) b(x)| \leq t\}$. Pick up $\eta>0$. Then we make a partition in the following way,

$$
\begin{aligned}
& \{x \in \Omega:|a(x) b(x)| \leq t\}=(\{x \in \Omega:|a(x) b(x)| \leq t\} \bigcap\{x \in \Omega:|a(x)| \geq \eta\}) \\
& \bigcup(\{x \in \Omega:|a(x) b(x)| \leq t\} \bigcap\{x \in \Omega:|a(x)|<\eta\}) .
\end{aligned}
$$

For the first subset, if $x$ in $\Omega$ satisfies both conditions $|a(x) b(x)| \leq t$ and $|a(x)| \geq \eta$ then $|b(x)| \leq \frac{t}{\eta}$ which means that

$$
\{x \in \Omega:|a(x) b(x)| \leq t\} \bigcap\{x \in \Omega:|a(x)| \geq \eta\} \subset\left\{x \in \Omega:|b(x)| \leq \frac{t}{\eta}\right\} .
$$

Clearly,

$$
\{x \in \Omega:|a(x) b(x)| \leq t\} \bigcap\{x \in \Omega:|a(x)|<\eta\} \subset\{x \in \Omega:|a(x)| \leq \eta\} .
$$

As a consequence, for $\eta=\sqrt{t}$,

$$
\begin{aligned}
& \operatorname{meas}\{x \in \Omega:|a(x) b(x)| \leq t\} \\
& \leq \operatorname{meas}\{x \in \Omega:|b(x)| \leq \sqrt{t}\}+\operatorname{meas}\{x \in \Omega:|a(x)| \leq \sqrt{t}\} .
\end{aligned}
$$

So, since $\varphi$ is a nondecreasing function on $[0, \gamma]$,

$$
\begin{aligned}
& \varphi(\operatorname{meas}\{x \in \Omega:|a(x) b(x)| \leq t\}) \\
& \leq \varphi\left(\operatorname{meas}\left\{x \in \Omega:|b(x)|^{2} \leq t\right\}+\operatorname{meas}\left\{x \in \Omega:|a(x)|^{2} \leq t\right\}\right) .
\end{aligned}
$$


But by 4$)$,

$$
\begin{aligned}
& \varphi(\operatorname{meas}\{x \in \Omega:|a(x) b(x)| \leq t\}) \leq \\
& C\left[\varphi\left(\operatorname{meas}\left\{x \in \Omega:|b(x)|^{2} \leq t\right\}\right)+\varphi\left(\text { meas }\left\{x \in \Omega:|a(x)|^{2} \leq t\right\}\right)\right] .
\end{aligned}
$$

By the previous proposition, $a^{2}$ and $b^{2}$ belong to $S_{\varphi}$ hence for some $c>0$ small enough,

$$
\begin{aligned}
& \int_{0}^{c} \frac{\varphi(\operatorname{meas}\{x \in \Omega:|a(x) b(x)| \leq t\})}{t} d t \leq \\
& C\left(\int_{0}^{c} \frac{\varphi\left(\operatorname{meas}\left\{x \in \Omega:|a(x)|^{2} \leq t\right\}\right)}{t} d t+\int_{0}^{c} \frac{\varphi\left(\operatorname{meas}\left\{x \in \Omega:|b(x)|^{2} \leq t\right\}\right)}{t} d t\right) .
\end{aligned}
$$

As a conclusion, $a b$ is in $S_{\varphi}$.

The next step is to find a new class of functions satisfying properties 1), 2), 3) and 4).

Proposition 4.3 Let $\varphi$ be a function defined on $[0, \gamma]$ for some $\gamma>0$ which satisfies 1), 2), 3) and

$$
\text { 4') } \left.\varphi \text { is a convex function on }[0, \gamma] \text { with } \limsup _{t \rightarrow 0^{+}} \frac{\varphi(2 t)}{\varphi(t)}<+\infty \text {. Then } \varphi \text { satisfies } 4\right) \text {. }
$$

Proof: Since $\varphi$ is convex on $[0, \gamma]$, for all $\alpha, \beta$ in $\left[0, \frac{\gamma}{2}\right], \varphi(\alpha+\beta) \leq \frac{\varphi(2 \alpha)+\varphi(2 \beta)}{2}$. It remains to prove that for all $t>0$ some enough, $\varphi(2 t) \leq C \varphi(t)$ for some $C>0$.

Always by convexity of $\varphi$, the function $t \mapsto \frac{\varphi(2 t)}{\varphi(t)}$ is continuous on $\left(0, \frac{\gamma}{2}\right]$ and bounded in a neighbourhood of zero (this function is nonnegative).

As a consequence, it is bounded on $\left(0, \frac{\gamma}{2}\right]$.

Proposition 4.4 The function $\varphi(t)=t(-\ln t)$ satisfies 1$), 2), 3)$ and $\left.4^{\prime}\right)$ for $\gamma=e^{-1}$. Moreover, the function $w(x)=\exp \left(-\frac{1}{|x|^{\frac{N}{2}}}\right)$ belongs to $S_{\varphi}$.

Proof: 1), 2), 3) are clear. For all $t \in\left(0, e^{-1}\right], \varphi^{\prime}(t)=\frac{1}{(-\ln t)}+\frac{1}{(-\ln t)^{2}}$ so $\varphi^{\prime}$ is an increasing function, hence, $\varphi$ is strictly convex. Clearly, $\lim _{t \rightarrow 0^{+}} \frac{\varphi(2 t)}{\varphi(t)}=2$.

With the estimate $\varphi(t) \leq t$ for $t \in\left[0, e^{-1}\right]$, we have

$$
\begin{aligned}
& \int_{0}^{e^{-1}} \frac{\varphi(\operatorname{meas}\{x \in \Omega:|w(x)| \leq t\})}{t} d t \leq \int_{0}^{e^{-1}} \frac{\operatorname{meas}\{x \in \Omega:|w(x)| \leq t\}}{t} d t \\
& =\int_{0}^{e^{-1}} \frac{\operatorname{meas}\left\{x \in \Omega:|x|^{\frac{N}{2}} \leq(-\ln t)^{-1}\right\}}{t} d t=C_{N} \int_{0}^{e^{-1}} \frac{1}{t(-\ln t)^{2}} d t<+\infty .
\end{aligned}
$$




\subsection{Orlicz spaces}

Let $A$ be an $N$-function [23]. When the derivative of $A$ called $\bar{a}$ is increasing, the $N$-functions $A$ and $\widehat{A}$ given by

$$
A(t)=\int_{0}^{t} \bar{a}(\tau) d \tau, \quad \widehat{A}(t)=\int_{0}^{t} \bar{a}^{-1}(\tau) d \tau,
$$

are said to be complementary. The Orlicz space connected to $A$ is denoted by $L_{A}(\Omega)$. If $E$ is a measurable set of positive measure, the Luxemburg norm is

$$
\|u\|_{L_{A}(E)}=\inf \left\{k>0: \int_{E} A\left(\frac{|u(x)|}{k}\right) d x \leq 1\right\}
$$

if the previous set is not empty and also we have a generalized version of Hölder's inequality,

$$
\left|\int_{E} u(t) v(t) d t\right| \leq 2\|u\|_{L_{A}(E)}\|v\|_{L_{\widehat{A}}(E)}
$$

Theorem 4.2 [25] Let $\Omega$ be a bounded domain of $\mathbb{R}^{N}$ having the cone property and $m p=N$ where $p>1$. Set $A(t)=\exp \left(t^{\frac{p}{p-1}}\right)-1$. Then there exists the imbedding $W^{m, p}(\Omega) \hookrightarrow L_{A}(\Omega)$.

Even if $A$ is an $N$-function, $M(t)=A(\sqrt{t})$ is not necessary an $N$-function but the quantity

$$
\|u\|_{L_{M}(E)}=\inf \left\{k>0: \int_{E} A\left(\sqrt{\frac{|u(x)|}{k}}\right) d x \leq 1\right\},
$$

is well defined for a measurable set $E$ of positive measure if $\int_{E} A\left(\sqrt{\frac{|u(x)|}{k}}\right) d x \leq 1$ for some positive $k$. With this extended notation, we have the following standart propositions :

Proposition 4.5 $\|v\|_{L_{A}(E)}^{2}=\left\|v^{2}\right\|_{L_{M}(E)}$ when the quantity in the left-hand side is well defined.

Proof: From the definition, $\|v\|_{L_{A}(E)}^{2}=\inf \left\{k>0: \int_{E} A\left(\frac{|v(x)|}{k}\right) d x \leq 1\right\}^{2}$. So,

$\|v\|_{L_{A}(E)}^{2}=\inf \left\{k>0: \int_{E} A\left(\sqrt{\frac{|v(x)|^{2}}{k^{2}}}\right) d x \leq 1\right\}^{2}$ gives

$\|v\|_{L_{A}(E)}^{2}=\inf \left\{k^{2}>0: \int_{E} A\left(\sqrt{\frac{|v(x)|^{2}}{k^{2}}}\right) d x \leq 1\right\}$ and

$\|v\|_{L_{A}(E)}^{2}=\left\|v^{2}\right\|_{L_{M}(E)}$. 
Proposition 4.6 If $B \leq A$ then $\|v\|_{L_{B}(E)} \leq\|v\|_{L_{A}(E)}$ when the quantity in the righthand side is well defined.

Proposition 4.7 If $E \subset F$ are two measurable sets of positive measure, $\|v\|_{L_{B}(E)} \leq\|v\|_{L_{B}(F)}$ when the quantity in the right-hand side is well defined.

Proposition 4.8 If $B$ is an $N$-function and $E$ a measurable set of positive measure then $\|v\|_{L_{B}(E)} \leq \frac{\|v\|_{L^{\infty}(E)}}{B^{-1}\left(\frac{1}{\text { meas }(E)}\right)}, \forall v \in L^{\infty}(E)$

Proposition 4.9 Let $f$ and $g$ be two increasing functions defined on a neighbourhood of $+\infty$ with $\lim _{x \rightarrow+\infty} f(x)=\lim _{x \rightarrow+\infty} g(x)=+\infty$. If $f(x) \leq g(x)$ for $x$ large enough then $f^{-1}(x) \geq g^{-1}(x)$ for $x$ large enough.

\subsection{Previous result for the second order case}

Theorem 4.2 ( [2]) Under assumptions (1.6) and (1.5) with $m=1$, if there exists a decreasing sequence $\left\{\alpha_{n}\right\}$ of positive real numbers such that

$$
\sum_{n=1}^{\infty} \frac{1}{\lambda_{1,2}\left(\alpha_{n}^{\frac{1-q}{2}}\right)}\left(\ln \left(\lambda_{1,2}\left(\alpha_{n}^{\frac{1-q}{2}}\right)\right)+\ln \left(\frac{\alpha_{n}}{\alpha_{n+1}}\right)+1\right)<+\infty
$$

then any weak solution of problem (1.1)-(1.3) vanishes in a finite time.

Acknowledgment. The authors are very grateful to Laurent Véron for useful discussions and valuable comments. Both authors have been supported by an INTAS grant through the Project INTAS 05-1000008-7921.

\section{References}

[1] V.A. Kondratiev and L. Véron, Asymptotic behaviour of solutions of some nonlinear parabolic or elliptic equations, Asymptotic Analysis 14 (1997), 117-156.

[2] Y. Belaud, B. Helffer, L. Véron, Long-time vanishing properties of solutions of sublinear parabolic equations and semi-classical limit of Schrödinger operator, Ann. Inst. Henri Poincaré Anal. nonlinear 18, 1 (2001), 43-68.

[3] Y. Belaud, A. E. Shishkov, Long-time extinction of solutions of some semilinear parabolic equations, J. Diff. Equations 238, (2007), 64-86.

[4] A. S. Kalashnikov, The propagation of disturbances of nonlinear heat equation with absorption, USSR Comput. Math. and Math Phys. 14 (1974), 70-85. 
[5] A. S. Kalashnikov, Instantaneous shinking of the support for solutions to certain parabolic equations and systems, Rend, Mt. Acc. Lincei. Serie 9, 8 (1997), 263-272.

[6] R. Kersner, Nonlinear heat conduction with absorption: space localization and extinction in finite time, SIAM J. Appl. Math. Vol. 436 (1983), 1274-1285.

[7] R. Kersner, F. Nicolosi, The nonlinear heat equation with absorption: effect of vaicable coefficients, J. Math. Anal. Appl. 170 (1992), 551-566.

[8] R. Kersner, A. Shishkov, Instantaneous shrinking of the support of energy solutions, J. Math. Anal. Appl. 198 (1996), 729-750.

[9] B. Straughau, Instability, nonexistence and weighted energy methods in fluid dynamics and related theories. Research Notes in Mathematics 74 (London: Pitman, 1982).

[10] L. E. Payne, Improperly posed problems in partial differential equations, SIAM Regional Conference Seeries in Applied Math, 22 (1975).

[11] B. F. Knerr, The behaviour of the support of solution of the equation of nonlinear heatconduction with absorption in one dimension. Trans. Amer. Math. Soc. 249 (1979), N 2, 409-424.

[12] Chen Xu-Yan, H. Matano, M. Mimura, Finite point extinction and continity of interfaces in nonlinear diffusion equations with strong absorption, J. Reine Angew. Math. 459:1 (1995), 1-36.

[13] Y. Belaud, Asymptotic estimates for a variational problem involving a quasilinear operator in the semi-classical limit, Annals of global analysis and geometry 26 : 271-313, 2004.

[14] A. E. Shishkov, Dead cores and instantaneous compactification of the supports of energy solutions of quasilinear parabolic equations of arbitrary order, Sbornik: Mathematics, 190:12, (1999), 1843-1869.

[15] J.I. Díaz and L. Véron, Local vanishing properties of solutions of elliptic and parabolic quasilinear equations, Trans. Amer. Math. Soc. 290 (1985), 787-814.

[16] E.H. Lieb, W. Thirring, Inequalities for the moments of the eigenvalues of the Schrödinger Hamiltonian and their relations to Sobolev Inequalities, In Studies in Math. Phys., essay in honour of V. Bargmann, Princeton Univ. Press, 1976.

[17] B. Helffer, Semi-classical analysis for the Schrödinger operator and applications, Lecture Notes in Math. 1336, Springer-Verlag, 1989.

[18] C. Bandle, I. Stakgold, The formation of the dead core in parabolic reactiondiffusion problems, Trans. Amer. Math. Soc. 286 (1984), 275-293. 
[19] A. Friedman, M. Herrero, Extinction properties of semilinear heat equation with strong absorption, J. Math. Anal. Appl. 124 (1987), 530-546.

[20] F. Bernis, Finite speed of propagation and asymptotic rates for some nonlinear higher order parabolic equations with absorption, Proc. Roy. Soc. Edinburgh 104A (1986), 1-19.

[21] Junjie Li, Qualitative properties for solutions of semilinear heat equations with strong absorption, J. Math. Anal. Appl. 281 (2003), 382-394.

[22] Junjie Li, Qualitative properties for solutions to semilinear heat equations with singular initial data, Electronic J. of Differ Equat. (2004), N 53, 1-12.

[23] M.A. Kranosel'skii and Ya.B. Rutickii, Convex Functions and Orlicz spaces, Noordhoff, Groningen, The Netherland, 1961.

[24] J.-L. Lions, Quelques méthodes de résolution des problèmes aux limites non linéaires, Dunod Gauthier-Villars, 1969.

[25] N.S. Trudinger, On imbeddings into Orlicz spaces and some applications, J. Math. Mech 17 (1967), 473-483. 